\title{
OA06-06 LB. Evidence of vaccine-induced changes in breakthrough HIV-I strains from the Step trial
}

M Rolland ${ }^{1}$, S Tovanabutra ${ }^{2}$, PB Gilbert ${ }^{3}$, E Sanders-Buell ${ }^{2}$, L Heath ${ }^{1}$, AC deCamp ${ }^{3}$, CC Magaret ${ }^{3}$, M Bose ${ }^{2}$, A Bradfield ${ }^{2}$, A O'Sullivan ${ }^{2}$, J Crossler ${ }^{2}$, W Deng ${ }^{1}$, H Zhao ${ }^{1}$, K Wong ${ }^{1}$, DN Raugi ${ }^{1}$, J Hural ${ }^{3}$, S Dubey ${ }^{3}$, N Frahm ${ }^{3}$, NL Michael ${ }^{4}$, J Shiver ${ }^{5}$, L Corey ${ }^{3}$, F Li ${ }^{3}$, SG Self ${ }^{3}$, J Kim ${ }^{4}$, S Buchbinder6, DR Casimiro ${ }^{5}$, MN Robertson ${ }^{5}$, MJ McElrath ${ }^{3}$, FE McCutchan ${ }^{4}$ and JI Mullins*1

Address: ${ }^{1}$ University of Washington, Seattle, WA, USA, ${ }^{2}$ US Military HIV Research Program, Rockville, MD, USA, ${ }^{3}$ Vaccine \& Infectious Dieseases Institute, Fred Hutchinson CRC, Seattle, WA, USA, ${ }^{4}$ US Military HIV Research Program, Rockville, MD, USA, ${ }^{5}$ Merck Research Laboratories, West Point, PA, USA and 'San Francisco Department of Health, San Francisco, CA, USA

* Corresponding author

from AIDS Vaccine 2009

Paris, France. 19-22 October 2009

Published: 22 October 2009

Retrovirology 2009, 6(Suppl 3):O42 doi:I0.I |86/I 742-4690-6-S3-O42

This abstract is available from: http://www.retrovirology.com/content/6/S3/O42

(c) 2009 BioMed Central Ltd.

\section{Background}

Vaccinations in the Step Study, designed to assess the efficacy of a T cell-based vaccine, were interrupted in 2007 after an interim analysis showed no evidence of efficacy.

\section{Methods}

To evaluate the impact of the vaccine on founder/breakthrough viruses, i.e., the viruses obtained from the earliest available specimen around the time of diagnosis, we obtained 0.5-14 HIV-1 whole genome sequences per individual from 40 vaccine and 28 placebo recipients who acquired HIV-1 infection as of December 2007.

\section{Results}

To assess whether the vaccine elicited $\mathrm{T}$ cells that exerted selective pressure on founder viruses, we identified known and likely class I MHC-restricted CTL epitopes in founder sequences based on each volunteer's HLA type. We calculated protein distances between epitopes in the vaccine and those in the founder sequences. Compared to the vaccine, epitopes from vaccine recipients exhibited more mutations than epitopes from placebo recipients $(p=0.02)$. Potential epitopes were then identified across all infected individuals and compared to HXB2. The epitopic distances from the combination of Gag, Pol and Nef sequences (included in vaccine) were significantly higher among vaccine vs. placebo recipients $(p=0.004)$. In contrast, there was no distinction between vaccine and placebo if epitopes from Env, Rev, Tat, Vif, Vpr, Vpu (not included in vaccine) were considered together $(\mathrm{p}=0.90)$. Lastly, 10 AA signature sites in Gag, Nef, Pol (but none in Env) were found to classify vaccine versus placebo status.

\section{Conclusion}

Our data indicate that founder viruses from vaccinees diverged further from the vaccine than viruses from placebo recipients at potentially immune reactive sites. This suggests that the vaccine may have blocked the outgrowth of specific HIV-1 variants that were the most similar to the vaccine sequence and/or elicited immune responses that may have driven specific mutations among vaccinees' viruses post-infection. 